\title{
Contrasting task demands alter the perceived duration of brief time intervals
}

\author{
THOMAS F. SAWYER \\ North Central College, Naperville, Illinois \\ PETER J. MEYERS \\ University of Iowa, Iowa City, Iowa \\ and \\ STACY J. HUSER \\ North Central College, Naperville, Illinois
}

\begin{abstract}
Four experiments were performed to assess the effects of task differences on duration judgments. Experiments 1 and 2 used the method of reproduction in prospective, within-subjects designs; their results supported previous research on the effects of task difficulty. Both experiments, using tasks that varied along somewhat different dimensions, found that subjects provided reproduction values that varied inversely with task difficulty. That is, while subjects tended to underreproduce across all tasks, the more difficult the task performed during the target interval, the greater the extent of the underreproduction. Experiments 3 and 4 used a modification of the reproduction method by placing demands upon the subjects during both the target interval and the reproduction phase of each trial; they demonstrated that the greater the degree of contrast between demands made by the task performed during the target interval and those made during reproduction, the less accurate the duration reproduction. The results are discussed in terms of the contextual and resource allocation models of duration estimation.
\end{abstract}

The contextual model of temporal experience (Block, $1989,1990)$ proposes a variety of factors that interact to influence psychological time. They include: (1) the particular kind of temporal behavior required of the observer (e.g., duration estimation, rhythm production); (2) the contents and qualities of the time period (e.g., its duration, the number of events); (3) the characteristics of the experiencer (e.g., personality factors, temporal outlook); and (4) the activities performed within the time period (e.g., problem solving, types of perceptual and/or cognitive requirements). The model has been a valuable heuristic, identifying a variety of relationships and interactions between time-relevant variables that it should be useful to explore, as well as assisting in resolving controversy that existed within the literature. This can be illustrated by an examination of research concerning both the effects of task demands on subjects' duration judgments and the way in which such effects are moderated by different instruction-based temporal outlooks (i.e., prospective versus retrospective paradigms).

A number of studies have shown that subjects' duration judgments for a given interval of time are affected by the

This research was supported by a faculty research grant awarded to T.F.S. by North Central College. Address reprint requests to T. F. Sawyer, Psychology Department, North Central College, 30 N. Brainard, P.O. Box 3063, Naperville, IL 60566-7063. types of task demands placed upon them during that interval. Very often, these research studies have employed a prospective duration-judgment paradigm (e.g., Burnside, 1971; Hicks, Miller, Gaes, \& Bierman, 1977; Smith, 1969; Zakay, 1993; Zakay, Nitzan, \& Glicksohn, 1983). In this paradigm, subjects are aware in advance that they will be required to make duration judgments. Most often, the subjects work at a particular task for some target interval and then render duration judgments either by stating their verbal estimate of the interval's duration (verbalestimation method) or by attempting to reproduce the interval by performing some behavioral response, such as saying "start" and "stop" as delimiters or pressing a button (reproduction method). Occasionally, as a means of obtaining duration judgments, studies within the prospective paradigm have used a production method, whereby the subjects are provided with a predefined duration (stated in minutes and/or seconds) and are asked to work at a task for what they believe to be that duration.

Other research has used a retrospective paradigm (e.g., Block, 1978; Predebon, 1988) in which the subjects, while they are engaged in the activity during the target interval, are unaware of the eventual duration judgments they will be asked to make. At the conclusion of the target interval, they are asked unexpectedly either to verbally estimate the duration of the interval or to reproduce its duration. It should be noted that some researchers have contrasted prospective and retrospective 
paradigms within the context of a single experiment (e.g., Brown, 1985; Hicks, Miller, \& Kinsbourne, 1976; Miller, Hicks, \& Willette, 1978; Zakay, 1989).

Those studies that have used a prospective paradigm and either verbal estimation or reproduction frequently report a significant negative relationship between task difficulty and subjects' duration judgments, with increases in task difficulty leading to correspondingly shorter duration judgments (see Block, 1990, or Zakay, 1993, for reviews). In contrast, while the results are somewhat mixed (e.g., Brown, 1985), those studies in which there has been found a positive relationship between variable task demands during the target interval and subjects' duration judgments have utilized the retrospective paradigm. Although such discrepant results can be easily accommodated within Block's (1989) contextual model, they are not readily predicted from it. To account for these conflicting results, Zakay (1989) drew upon the contextual model, as well as upon a previous model of Thomas and Weaver (1975), in formulating the resource allocation model, which proposes that retrospective and prospective paradigms involve fundamentally different processes. Specifically, it hypothesizes that long-term memory (LTM) mechanisms, such as those previously suggested within the storage-size (Ornstein, 1969) and change-segmentation (Poynter, 1983, 1989) models, might best account for results within a retrospective paradigm, while a model focusing on attention and short-term memory (STM) mechanisms might best account for findings within a prospective paradigm.

Within a retrospective paradigm, for example, subjects are unaware of the upcoming request for a duration judgment while they are engaged in a task during the target interval, and they must necessarily receive instructions on providing the dependent measure after that interval. The required processing of such instructions prior to producing the verbal estimate or reproduction would occupy their working memory, and would suggest that subjects must develop their duration judgments from information about the target interval that was stored in LTM during that interval. In this case, increasing task difficulty might be expected to increase the amount of processing and storage. Further, the assumption that subjects base their duration judgments upon amount processed/stored (e.g., Ornstein, 1969) would lead to the expectation of a positive relationship between task difficulty and duration estimates, which is consistent with the general finding noted previously (Block, 1990; Zakay, 1993). Alternatively, in a prospective paradigm, since subjects are aware of the impending duration judgment they will be asked to make, they are likely to direct some attention toward time-relevant information while engaged in the task during the target interval, and thus largely base their eventual verbal estimate or reproduction upon information available in STM. Any increases in the difficulty of the task performed during the target interval would be expected to detract attention from such temporal monitoring and, in the model de- scribed by Zakay (1989), should result in a shorter duration estimate. Such a prediction, in contrast to that for a retrospective paradigm, agrees with the previously described negative relationship between task demands and duration judgments consistently found within a prospective paradigm (Block, 1990; Zakay, 1993).

Within the context of a prospective paradigm, the present study investigated the ability of the attentional model (Zakay, 1989) to account for the effects of task manipulations upon duration judgments. In Experiments 1 and 2 , the reproduction method was used in an attempt to establish the generalizability to other sets of tasks of the reported negative relationship between difficulty and duration judgments. Experiments 3 and 4 employed a modified version of the reproduction method which placed demands upon subjects during both the target interval and the reproduction phase of a trial. In the latter two experiments, the subjects encountered trials in which the tasks of the target interval and reproduction phase were comparable, as well as other trials with contrasting tasks. The attentional model would predict (1) minimal error for comparable tasks and increasing error with increasing task contrast and (2) with contrasting tasks, negative error (i.e., underreproduction) when the task of the target interval is more difficult than that of the reproduction phase, and positive error (i.e., overreproduction) when the task of the target interval is less difficult than that of the reproduction phase.

\section{EXPERIMENT 1}

As well as emphasizing the importance of STM processes in prospective duration estimation, Zakay's (1989) attentional model proposes the presence of a cognitive timer. The model states that during a target interval, the cognitive timer accumulates subjective time units (STUs), and that the eventual duration estimates made by subjects are a function of the number of such units accumulated during the interval. In addition, the cognitive timer is presumed to require attentional resources of the subject; furthermore, the accumulation of STUs is held to be a function of the amount of attention devoted to the cognitive timer during the interval. When tasks that require attention must be performed by the subject during the target interval, a concomitant reduction in attention devoted to the cognitive timer would be expected. Thus, as task demands increase, a reduction in the accumulation of STUs should occur, resulting in shorter duration judgments by the subjects.

Experiment 1 employed the reproduction method, and was an attempt to replicate previous research concerned with task effects on duration estimation using a set of tasks that involved variable levels of motor, perceptual, and cognitive components.

\section{Method}

Subjects. A total of 15 students ( 7 male, 8 female) were recruited from introductory psychology classes, in partial fulfillment of 
course requirements, for what was described as an experiment in time perception. In this and all experiments to be reported, the subjects were tested individually.

Task descriptions. The subjects were exposed to the following four types of paper-and-pencil tasks: (1) a motor task, wherein the subjects were given a page of preprinted diagonal marks, on which they were required to make their own marks, turning them into $X \mathbf{s}$ (designated "make $X \mathrm{~s}$ "); (2) a perceptual identification task, requiring subjects to search through rows of random letters, crossing off the letter $A$ wherever it occurred ("cross $A$ s"); (3) a task in which the subjects were exposed to a sheet containing four-letter anagrams which they were to try and solve, writing the correct words in spaces provided ("anagrams");" and (4) a "task" in which the subject was exposed to a blank sheet, which specified an empty time interval that had no task demands ("no task").

That these tasks represent different levels of difficulty was verified using a concurrent task procedure with an independent group of 16 subjects, who performed each of the tasks for $18 \mathrm{sec}$. Two seconds into the interval, they heard a two-digit number, which was the signal for them to count by $3 \mathrm{~s}$ from that number (for example, if the two-digit number was 29 , the subject was required to count " $32,35,38,41,44, \ldots$ " The subjects had been instructed to make the paper-and-pencil task the primary one and not to allow counting to interfere with its performance. Significant variation across the paper-and-pencil tasks was found in the number of values that the subjects were able to accurately count off, suggesting differences in the tasks' difficulty that were as expected; that is, from easiest to most difficult, the different tasks were ordered: no task ( $M=11.0$ accurate counts); make $X \mathrm{~s}(M=9.6)$; cross $A \mathrm{~s}(M=$ 7.7); and anagrams $(M=4.6)$. Comparable results were provided by another group of subjects, who worked on the paper-and-pencil tasks while engaged in a concurrent digit-recall task.

Duration measurement. For each trial, the target interval was initiated by a $0.1-\mathrm{sec}$ tone from a Lafayette Instruments Tone Generator, Model 58025, that signaled the subject to begin working on the task for that trial. The interval was terminated by a second signal, at which point the subject placed the task sheet aside and immediately attempted to reproduce the duration of the target interval by depressing a telegraph key for a period equal to the length of time she or he had been working on the task. Pressure on the telegraph key activated a digital stopclock, providing the dependent measure for the trial. The actual objective duration of the target interval was controlled by the experimenter via an event timer accurate to $0.1 \mathrm{sec}$.

Procedure. An 18-sec practice trial with each of the tasks (in the order cross $A \mathrm{~s}$, make $X \mathrm{~s}$, anagrams, and no task) acquainted each subject with each task and the reproduction method. Following the practice trials, each subject was exposed to each of the four tasks for each of three durations $(14,18$, and $22 \mathrm{sec})$, yielding a total of 12 experimental trials. For a particular subject, the trials were presented according to one of eight randomly generated orders, with an intertrial interval (ITI) of 15-20 sec.

\section{Results}

Experiment 1 constituted a 4 (task) $\times 3$ (interval) within-subjects factorial design, and the results are graphically displayed in Figure 1. As expected, the subjects showed a tendency to underestimate the target interval across all interval durations, with the degree of underestimation increasing with greater task demands. An analysis of variance (ANOVA) performed on the reproduction values across all trials showed a significant effect for task $[F(3,42)=41.62, p<.001]$, as well as for target-interval duration $[F(2,28)=62.85, p<.001]$, while their interaction did not reach significance $[F(6,84)=1.89, p>.09]$. Pairwise comparisons for lev-

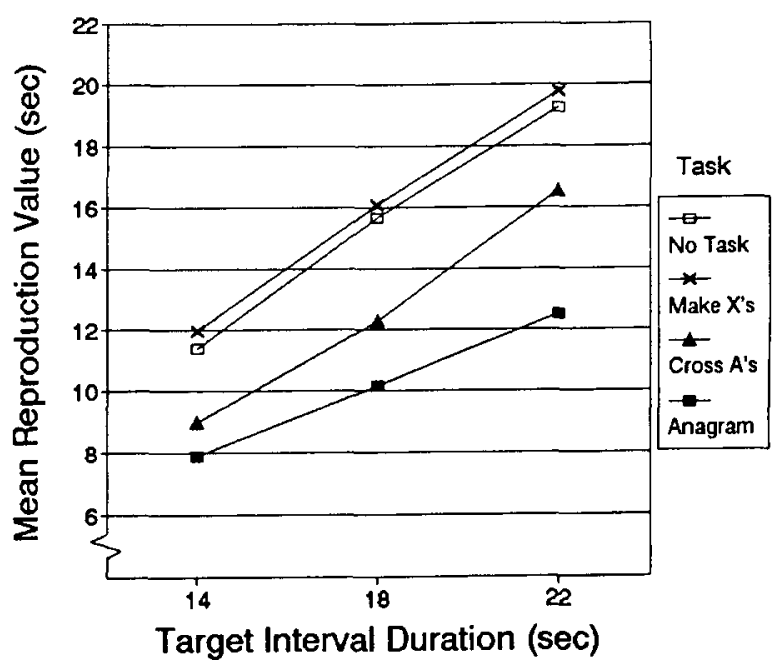

Figure 1. Results of Experiment 1. Effects of task on reproduction time estimates across the three different time intervals.

els of task difficulty collapsed across intervals were performed using the Newman-Keuls procedure. These comparisons demonstrated that subjects gave significantly lower reproduction values after the anagrams task than they did following any of the other tasks [no task, $p<.01$; make $X \mathrm{~s}, p<.01$; cross $A \mathrm{~s}, p<.05$ ]. Likewise, following cross $A$ s, the subjects provided significantly lower reproduction values than they did following both no task $(p<.01)$ and make $X \mathrm{~s}(p<.01)$. Finally, reproduction values obtained from subjects after no task did not differ significantly from those obtained after make $X \mathrm{~s}(p>.20)$.

\section{EXPERIMENT 2}

In a second experiment, we sought to extend the results of Experiment 1 to a set of tasks different from those used in Experiment 1 but similar to those used in earlier research (Zakay, 1993; Zakay \& Fallach, 1984), in order to confirm the effectiveness of our methodology. Other than the types of task used, this experiment was methodologically comparable to Experiment 1.

\section{Method}

Subjects. Fourteen students ( 7 male, 7 female) were recruited from introductory psychology classes, in partial fulfillment of course requirements, for what was described as an experiment in time perception.

Task descriptions. In the same way as in Experiment 1, a set of four tasks was used, this time, however, varying in the degree of response interference. They required the subjects either (1) to simply read out loud lists of color names (red, green, blue, purple, yellow, and black), all of which were printed in black ink (designated "black word"); (2) to examine groupings of 3-6 asterisks printed in colored ink (red, green, blue, purple, yellow, or black), and to identify the color of each group of asterisks ("asterisk"); (3) to identify the color of ink in which several unrelated words (key, cabin, song, friend, pencil, and glass) were printed ("other word"); (4) to examine color names (red, green, blue, purple, yellow, and 
black) printed in incongruent colored ink and to name the color of the ink ("Stroop").

Significant prior research (see Dyer, 1973, or Jensen \& Rohwer, 1966, for reviews) has demonstrated that the Stroop task is more demanding than the black-word task, with the asterisk and otherword tasks being intermediate. Similar sorts of tasks have been used successfully by Zakay and colleagues in time research (e.g., Zakay, 1993; Zakay \& Fallach, 1984).

Procedure. The procedures for making duration measurements as well as task presentations were identical to those of Experiment 1 . Practice trials with each task were given, following which the subjects were exposed to each of the four tasks for each of three durations $(14,18$, and $22 \mathrm{sec})$, for a total of 12 experimental trials.

\section{Results}

The results are plotted as a function of task and target-interval duration in Figure 2. A 4 (task) $\times 3$ (interval) within-subjects ANOVA performed on the reproduction values across trials showed a significant effect for task $[F(3,39)=17.20, p<.001]$, as well as for interval duration $[F(2,26)=66.02, p<.001]$, while their interaction did not reach significance $[F(6,78)<1]$. While the mean reproduced durations for each task, collapsed across levels of interval, were ordered as expected (i.e., Stroop lowest, other word and asterisk intermediate, and black word highest), the only pairwise difference to reach statistical significance was that between duration reproduction following the Stroop task and that following the black-word task $(p<.05)$.

\section{Discussion of Experiments 1 and 2}

The results of Experiments 1 and 2 were consistent with previous research concerning the effects of task demands on duration estimation and are compatible with the expectations of the attentional model. For simpler tasks (e.g., black word and make $X$ s), the subjects could devote extensive attention to the cognitive timer both during the target interval and while engaged in reproduction. As a result, the pace at which STUs accumu-

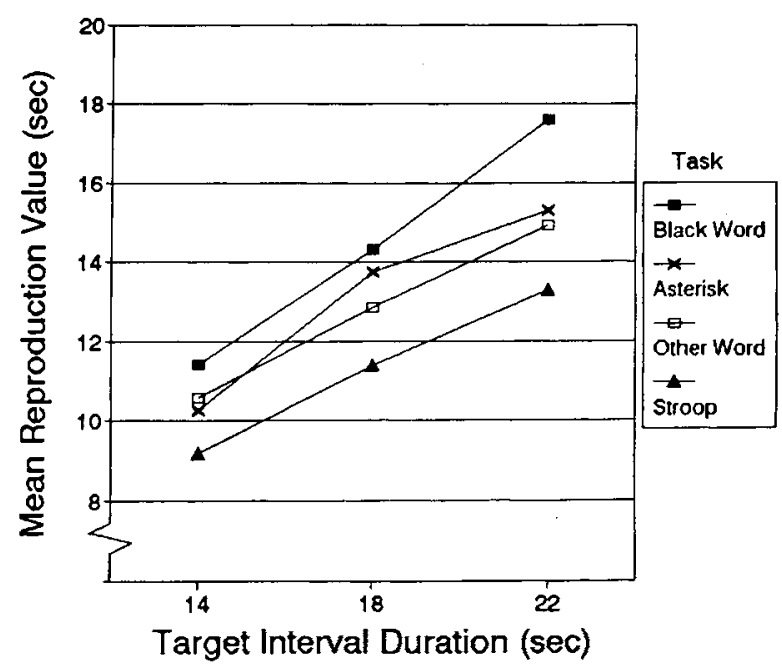

Figure 2. Results of Experiment 2. Effects of task on reproduction time estimates across the three different time intervals. lated during the reproduction phase was not very different from the pace during the target interval, resulting in relatively accurate reproduction values. However, for more difficult tasks (e.g., Stroop and anagrams), attentional resources were diverted away from the cognitive timer toward the task demands of the target interval. Finally, without a task during the reproduction phase, the STUs accumulated much more rapidly, leading to significant underreproduction of the target-interval duration.

Although the results of Experiment 1 and 2 are consistent with the attentional model, it is reasonable to question the necessity of proposing the presence of a cognitive timer whose functioning is influenced by task demands placed upon the subject. It would seem more parsimonious to suggest that subjects simply adopt overt, behavioral strategies designed to monitor the duration of the target interval - such as counting silently to themselves-and that when increased task demands are placed upon them, they are less able to utilize such methods and must resort to less reliable methods of keeping time. However, while it might predict more variable duration judgments with increases in task difficulty, such a hypothesis based upon behavioral strategies would not predict the consistent finding of increasing underestimation of duration with increasing task difficulty (e.g., Burnside, 1971; Hicks et al., 1977; Smith, 1969; Zakay, 1989; Zakay et al., 1983), as the attentional model does.

\section{EXPERIMENT 3}

Invariably, previous studies that have used the reproduction method to obtain duration estimates-including our Experiments 1 and 2 - have placed no task demands on the subject while he or she is engaged in reproducing the target interval. As a result, the more mindless the task in which a subject engages during the interval (e.g., no task or make $X \mathrm{~s}$ ), the more similar it is to the lack of demands placed upon the subject during reproduction. However, when a subject is faced with a difficult task during the target interval (e.g., anagrams or Stroop), there is significant contrast with the lack of demands that exist when the subject is involved in reproducing the duration of the interval.

In Experiment 3, we sought to investigate the effects on duration estimation of experimentally manipulating the contrast that existed between the task demands placed upon a subject during the target interval and those made during the reproduction phase of a trial. That is, for each trial, after working on one task during the target interval, the subjects were asked to work at a second task for what they believed to be the same duration as they had worked during the target interval. On some trials, the task demands for the two phases were the same; in others, the second task was of greater or lesser difficulty than the first.

The attentional model would predict that accuracy of duration estimation would be a function of the degree of concordance between the two tasks. Although subjects who engage in difficult tasks during a target interval 
greatly underestimate duration when no demands are placed upon them during reproduction--as was found in Experiments 1 and 2-the attentional model would predict that those who performed a difficult task during a target interval would provide relatively accurate duration estimates when they engaged in a similarly demanding task during reproduction. Simply put, if the tasks performed during the target interval and reproduction phase are comparable, the amount of attention devoted to the cognitive timer in each phase should be comparable, and the accumulation of STUs should proceed at a similar pace. Hence, the subject should work at the task during reproduction for approximately the same amount of time as she or he did during the target interval. When subjects engage in a simple task during the target interval but then perform a more difficult task during reproduction, however, significant overreproduction of the target interval should occur. This would result from the tendency for the accumulation of STUs to occur quickly during the target interval but more slowly during the reproduction phase of the trial. Finally, underreproductions of the target interval are predicted when the task that subjects work at during reproduction is simpler than the task they perform during the target interval.

\section{Method}

Subjects. Fourteen students ( 8 male, 6 female) were recruited from introductory psychology classes, in partial fulfillment of course requirements, for what was described as an experiment in time perception.

Task descriptions. The subjects were exposed to the same tasks that were described for Experiment 1, with the exception that the no-task condition was eliminated, as it was found not to produce results significantly different from the make $X$ s task.

Duration measurement. As in the previous experiments, the target interval for each trial was initiated and terminated by an auditory signal, between which the subject worked on the given task (designated as Task 1). At the second signal, the subject placed the first task sheet aside and picked up a second task sheet. The subject was instructed to try and work on this task (designated as Task 2) for a period equal to the length of time she or he had been working on Task 1. A device was constructed consisting of a springloaded writing surface $(38 \times 52 \mathrm{~cm})$ resting on a telegraph key that was connected to a digital stopclock. Pressure anywhere on the surface would depress the key, activating the stopclock. Subjects were instructed to keep one hand on the writing surface whenever they performed a task and to remove both hands when the task had been completed. This ensured consistent clock operation during the target interval and reproduction phase of each trial, and only during those times.

Procedure. Two 18-sec practice trials, one in which both tasks were identical and one in which the tasks differed, ensured each subject's exposure to each of the three task types. Following this, each subject was exposed to all combinations in the 3 (Task 1) $\times$ 2 (interval) $\times 3$ (Task 2 ) design, such that on any particular trial, she or he was exposed to one of three tasks during the target interval (make $X \mathrm{~s}$, cross $A \mathrm{~s}$, or anagrams) for one of two different durations ( 14 or $22 \mathrm{sec}$ ), and then performed one of the three tasks during reproduction. This yielded 18 experimental trials; as in Experiments 1 and 2, the trials were presented according to one of eight randomly generated orders, with an ITI of 15-20 sec.

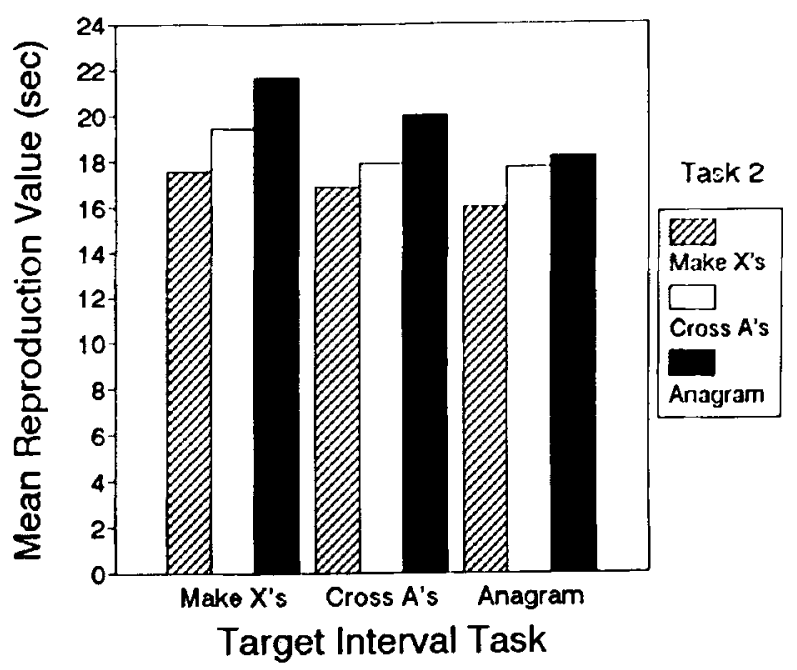

Figure 3. Results of Experiment 3. Task contrast effects on reproduction time estimates collapsed across the two different time intervals. The average of the two target intervals is $18 \mathrm{sec}$, and this figure represents accurate reproduction.

\section{Results}

A within-subjects ANOVA (Task $1 \times$ Task $2 \times$ interval) performed on the reproduction values across all trials provided a main effect for Task $1[F(2,26)=6.62$, $p<.01]$, a main effect for Task $2[F(2,26)=11.57, p<$ $.001]$, and the obvious main effect for interval $[F(1,13)=$ $140.91, p<.001]$. None of the two-way interactions approached significance (all $p \mathrm{~s}>.20$ ), and neither did the three-way interaction $(p>40)$. Figure 3 provides a graphical description of the results collapsed across the two interval durations. The most clear and concise way to describe these data is as follows: (1) The subjects tended to closely reproduce target intervals when they performed the same type of task during reproduction (i.e., Task 2) as they did during the target interval; (2) reminiscent of the findings of Experiments 1 and 2, working at simpler tasks during reproduction than during the target interval (e.g., anagrams as Task 1 and make $X$ s as Task 2) led to underreproduction of that interval; and (3) working at more difficult tasks during reproduction than during the target interval (e.g., make $X \mathrm{~s}$ as Task 1 and anagrams as Task 2) led to overreproduction.

\section{Discussion}

The results of Experiment 3 are consistent with the attentional model, which suggests that prospective duration judgments made by subjects are based upon the accumulation of STUs, whose rate varies as a function of the task performed, so that as long as the relative difficulties of the tasks are equivalent, the reproduction values should be close to the objective duration of the target interval. With increasing contrast between the tasks of the target interval and the reproduction phase, the attentional model predicted that, depending upon the na- 
Table 1

Expected Proportional Errors (EPE) and Actual Proportional Errors (APE) for Each Cell of Experiment 3

\begin{tabular}{|c|c|c|c|}
\hline \multirow[b]{3}{*}{ Task 1} & \multicolumn{3}{|c|}{ Task 2} \\
\hline & Make $X s$ & Cross $A \mathrm{~s}$ & Anagrams \\
\hline & EPE/APE & EPE/APE & EPE/APE \\
\hline Make $X \mathrm{~s}$ & $.000 /-.029$ & $+.268 /+.095$ & $+.563 /+.209$ \\
\hline Cross $A \mathrm{~s}$ & $-.211 /-.061$ & $.000 /+.009$ & $+.233 /+.152$ \\
\hline Anagrams & $-.360 /-.130$ & $-.189 /-.035$ & $.000 /+.046$ \\
\hline
\end{tabular}

Note-APE represents the proportional error of the reproduction values from the objective duration of the target intervals.

ture of the contrast, the reproduction values would be over or under the objective duration; this prediction was also confirmed in Experiment 3.

In this regard, an attempt was made to use the results of Experiment 1 to generate post hoc, quantitative predictions of errors in duration reproduction across the cells of Experiment $3 .^{2}$ Specifically, the reproduction values of Experiment 1 were converted to proportions by dividing by the appropriate interval duration. Collapsing across interval durations and subjects yielded mean reproduction proportions (MRP) for the relevant tasks (make $X \mathrm{~s}, \mathrm{MRP}_{\mathrm{MX}}=.886$; cross $A \mathrm{~s}, \mathrm{MRP}_{\mathrm{CA}}=.699$; anagrams, $\mathrm{MRP}_{\mathrm{AG}}=.567$ ). Finally, expected proportional errors (EPE) were calculated for each cell of Experiment 3 by first dividing the MRP for Task 1 (i.e., the task performed during the target interval) by the MRP for Task 2 (i.e., the task performed during reproduction) and then subtracting 1.0 to form the EPE. ${ }^{3}$ Positive values for EPE represent an expected overreproduction of the target interval, while negative values represent an expected underreproduction. Table 1 displays the results of the calculations, along with the actual proportional errors (APE) for each of the cells in Experiment 3.

Although the errors that occur with increasing task contrast do not increase as much as expected, there clearly is agreement between the EPEs generated from the results of Experiment 1 and the APEs for Experiment 3. A Spearman coefficient of rank correlation (Gibbons, 1976), calculated to assess the degree of relationship between EPE and APE across cells, was highly significant $(R=+.967, p<.001)$.

\section{EXPERIMENT 4}

An alternative to the attentional model that could explain some aspects of Experiment 3 lies in the possibility that during the reproduction phase, subjects simply made an effort to produce the same amount of "work" they had accomplished during the target interval (e.g., cross the same number of $A \mathrm{~s}$, solve the same number of anagrams). This might explain the close correspondence between the reproduction values and the objective duration of the target interval on those trials in which the tasks were equivalent during those phases. However, it is not clear how such a "work-production" hypothesis might account for the specific over- and underrepro- ductions of the target interval that were found when contrasting task demands were presented in the two phases of a trial.

Experiment 4 was an attempt to more clearly refute the work-production hypothesis, as well as to further evaluate the ability of the attentional model to account for task-complexity effects on duration judgments. The procedures of this experiment were quite similar to those of Experiment 3, in that the subjects were faced with one of three tasks during the target interval (i.e., make $X \mathrm{~s}$, cross $A \mathrm{~s}$, or anagrams) and were required to reproduce this time interval while working at another task. However, in the reproduction phase of this experiment, the subjects were faced with distinctively different tasksnamely, the black-word and Stroop tasks of Experiment 2 . Since no trials would have equivalent tasks during both target interval and reproduction phase, the work-production hypothesis would not lead to clear-cut expectations concerning the resulting reproduction values. The attentional model, however, predicted results not unlike those of Experiment 3; that is, regardless of the specifics of a particular task, the greater the demands it places on the subject, the slower the STUs should accumulate, while for less difficult tasks, the STUs should accumulate more rapidly. Thus, when subjects work at anagrams during the target interval and then work at the Stroop task during reproduction-both of them difficult tasks - one might expect somewhat similar levels of attention to the cognitive timer, and hence relatively accurate reproductions of the target interval on such trials. Furthermore, when subjects are faced with contrasting tasks (e.g., make $X$ s during target interval and Stroop task in reproduction, or anagrams during target interval and black word during reproduction), it was predicted that, depending upon the nature of the contrast, they would generate over- or underreproductions of the target-interval duration, as was found in Experiment 3.

\section{Method}

Subjects. Fifteen students ( 6 female, 9 male) were recruited from introductory psychology classes, and served in partial fulfillment of course requirements, for an experiment in time perception.

Task descriptions. The tasks used during target intervals were the same as those in Experiment 3-namely, make $X \mathrm{~s}$, cross $A$ s, and anagrams. During the reproduction phase of a trial, either the black-word or the Stroop task of Experiment 2 was used.

Procedure. Initially, a subject was shown sample task sheets for the make $X \mathrm{~s}$, cross $A \mathrm{~s}$, and anagram tasks she or he would work on during the target interval and was given instructions on how to perform each task. The subject was then shown sample sheets for the black-word and Stroop tasks on which she or he would work during the reproduction phase of a trial and was instructed on how to do each. The subject was then exposed to two 18 -sec practice trials. For both of these trials, the subjects were given the cross $A \mathrm{~s}$ task during the target interval, and during the reproduction phase performed the black-word task for one of the trials and the Stroop task for the other, counterbalanced across subjects.

Following practice, each subject was exposed to all combinations in the 3 (Task 1 ) $\times 2$ (interval) $\times 2$ (Task 2 ) design, such that on any particular trial, she or he was exposed to one of three tasks during the target interval (make $X \mathrm{~s}$, cross $A \mathrm{~s}$, or anagrams) for one 
Table 2

Subjects' Ratings of Task Difficulty in Experiment 4

\begin{tabular}{lccccc}
\hline & \multicolumn{5}{c}{ Task } \\
\cline { 2 - 6 } & Make $X \mathrm{~s}$ & Cross $A \mathrm{~s}$ & Anagrams & Black Word & Stroop \\
\hline Mean & 1.267 & 2.867 & 6.733 & 2.067 & 7.533 \\
SEM & .153 & .389 & .408 & .345 & .350 \\
\hline
\end{tabular}

Table 3

Mean Reproduction Values for Each Task 2 Condition as a Function of Interval and Collapsed Across Task 1 Conditions

\begin{tabular}{lccc}
\hline & \multicolumn{3}{c}{ Target Interval Duration } \\
\cline { 2 - 4 } Task 2 & $14 \mathrm{sec}$ & $22 \mathrm{sec}$ & Both Durations \\
\hline Black word & $13.47 \mathrm{sec}$ & $18.15 \mathrm{sec}$ & $15.81 \mathrm{sec}$ \\
Stroop task & $15.67 \mathrm{sec}$ & $23.49 \mathrm{sec}$ & $19.58 \mathrm{sec}$ \\
Both tasks & $14.57 \mathrm{sec}$ & $20.82 \mathrm{sec}$ & \\
\hline
\end{tabular}

of two different interval durations ( 14 or $22 \mathrm{sec}$ ), and then performed one of two tasks during reproduction (black word or Stroop). This generated 12 experimental trials, which, as in previous experiments, were presented according to one of eight randomly generated orders, with an ITI of 15-20 sec.

Following completion of the experimental trials, the subjects were asked to consider all five of the different tasks they had performed, and to verbally rate each on a scale of $1-10$, in which 1 meant "very, very simple" and 10 meant "very, very difficult." While it is recognized that these ratings are self-reports and, as such, should be viewed cautiously, they were gathered in order to gain another empirically based measure of differences in task difficulty.

\section{Results}

Table 2 provides a summary of the subjects' ratings of task difficulty. A one-way, within subjects ANOVA performed on these data revealed significant differences among the tasks $[F(4,56)=69.78, p<.001]$. Pairwise comparisons (Newman-Keuls) showed no difference between anagram and Stroop tasks, but both of these were rated as significantly more difficult than each of the other three tasks (all $p s<.01$ ). In addition, make $X \mathrm{~s}$ and black-word ratings were not significantly different. Further, although cross $A$ s was not rated as being different from black word, its mean rating was significantly higher than make $X \mathrm{~s}(p<.05)$.

A within-subjects ANOVA (Task $1 \times$ Task $2 \times$ interval) performed on the reproduction values across experimental trials provided a main effect for Task $1[F(2,28)=$ $5.72, p<.01]$, a main effect for Task $2[F(1,14)=32.90$, $p<.001]$, and the obvious main effect for interval $[F(1,14)=124.68, p<.001]$. In addition to these main effects, the two-way interaction between the task performed during the reproduction phase (i.e., Task 2) and the objective duration of the target interval was significant $[F(1,14)=17.83, p<.01]$. No other two-way interactions approached significance $(F \mathrm{~s}<1)$, and neither did the Task $1 \times$ Task $2 \times$ interval interaction $(F<1)$.

Analysis of the simple main effects of Task 2 at the two different interval durations (i.e., $14 \mathrm{sec}$ and $22 \mathrm{sec}$ ) showed that on trials in which Task 2 was black word, the subjects' reproduction values were significantly lower than they were on trials with Stroop as Task 2, fol- lowing both the 14-sec interval $[F(1,14)=17.57, p<$ $.01]$ and the 22-sec interval $[F(1,14)=103.21, p<$ $.001]$. Further, as indicated in Table 3 , the difference between the mean reproduction values for black-word trials versus Stroop trials was nearly two and one-half times greater for the 22-sec target interval than it was for the 14-sec interval. That is, the relative effect of Task 2 appears to have increased as the duration of the target interval increased from 14 to $22 \mathrm{sec}$, suggesting the basis for the significant Task $2 \times$ target interval interaction.

Regardless, the presence of this interaction does not obscure the previously cited significant main effects of Task 1 and Task 2, which replicate those found in Experiment 3 . In addition, examination of Figure 4, which portrays the reproduction values as a function of Task 1 and Task 2, collapsed across the two target-interval durations, indicates the similarity between the results of Experiments 3 and 4. In both experiments, for example, when subjects worked on tasks which they subsequently rated as of comparable difficulty (e.g., anagrams followed by Stroop), they tended to provide reproduction values relatively close to the actual duration of the target interval. When a more difficult task was performed during the reproduction phase than had been performed during the target interval (e.g., make $X$ s followed by Stroop), subjects tended to overreproduce the target interval. Additionally, when the tasks contrasted, such that the one performed during the target interval was the more difficult (e.g., anagrams followed by black word), the well-replicated tendency toward underreproduction was found.

As was done following Experiment 3, an attempt was made to generate post hoc, quantitative predictions of errors in duration reproduction for the cells created by combinations of Task 1 and Task 2. Computation of the

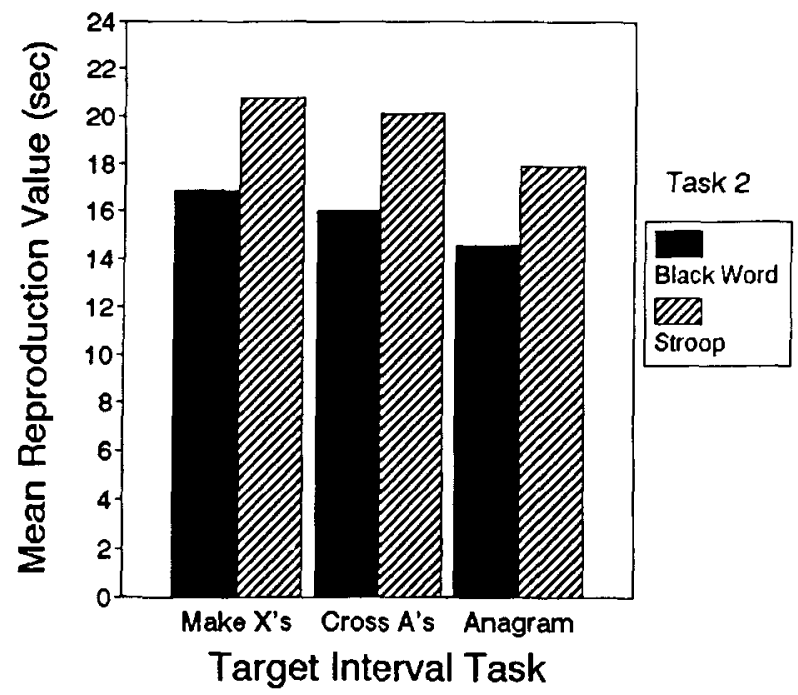

Figure 4. Results of Experiment 4. Task contrast effects on reproduction time estimates collapsed across the two different time intervals. The average of the two target intervals is $18 \mathrm{sec}$, and this figure represents accurate reproduction. 
Table 4

Expected Proportional Errors (EPE) and Actual Proportional Errors (APE) for Each Cell of Experiment 4

\begin{tabular}{lcc} 
& \multicolumn{3}{c}{ Errors (APE) Tor Each Cell of Experiment } \\
& Black Word & Stroop \\
Task 1 & EPE/APE & EPE/APE \\
\hline Make $X \mathrm{~s}$ & $+.103 /-.066$ & $+.415 /+.153$ \\
Cross $A \mathrm{~s}$ & $-.130 /-.108$ & $+.117 /+.117$ \\
Anagrams & $-.294 /-.191$ & $-.094 /-.006$ \\
\hline
\end{tabular}

Note-APE represents the proportional error of the reproduction values from the objective duration of the target intervals.

EPEs for each cell required calculation of MRPs for the black-word task $\left(\mathrm{MRP}_{\mathrm{BW}}=.803\right)$ and for the Stroop task $\left(\mathrm{MRP}_{\mathrm{ST}}=.626\right)$, as well as use of MRPs previously generated for make $X \mathrm{~s}$, cross $A \mathrm{~s}$, and anagrams. Table 4 presents the EPEs for each cell, along with the corresponding APEs. As in Experiment 3, there is generally good agreement between the expected and actual values. A Spearman coefficient of rank correlation between EPE and APE for the six cells was significant $(R=+.943$, $p<.01$ ).

\section{Discussion}

The results of Experiment 4 replicate and extend those of Experiment 3. In particular, the accurate duration reproduction following subjects' exposure to tasks that they rated as similar in relative difficulty but distinctively different in actual behavioral demands (e.g., anagrams followed by Stroop) provides strong support for the attentional model of duration estimation in prospective paradigms. In addition, exposing subjects to tasks of contrasting difficulties during the target interval and the reproduction phase yielded predictable over-or underreproductions, replicating those found in Experiment 3.

\section{GENERAL DISCUSSION}

The results of the present experiments replicate and extend the results of previous research concerned with the influence of task variation on prospective duration estimation (e.g., Smith, 1969; Zakay, 1993). In particular, the results are consistent with the attentional model of prospective duration estimation as described within the context of Zakay's (1989) resource allocation model. However, despite the support provided by these data, a variety of aspects of this model must be clarified in order for it to become a more effective explanatory model. As others have discussed (e.g., Block, 1990; Zakay, 1990), certain aspects of the attentional model are somewhat fuzzy. For example, the nature of the cognitive timer and the hypothesized accumulation of STUs may provide for accurate prediction, but fall short of furnishing a clear explanation of the experimental results. In addition, the notion that subjects can devote attention to temporal processing (i.e., to the cognitive timer) must be more clearly articulated (Block, 1990). Zakay's (1990) suggestions that "attention to time is the energy that activates the counter" (p. 61) and that "the 'temporal information' is not external but rather an internal input to the time processor" (p. 61) do not seem to put the issue to rest.

The results of these experiments also provide evidence concerning the importance of contextual factors in temporal experience (Block, 1989). Within the scope of the contextual model of temporal experience, it seems reasonable that a person's efforts to reproduce a target interval would be affected not only by the activities in which she or he was engaged during the target interval, but also by contextual features that existed during reproduction. This has not gone unnoticed by experienced coaches and/or performance instructors who recognize the importance of practicing coordinated, time-sensitive behaviors within a context close to that in which the activity will be eventually performed. The timing between quarterback and wide receiver, or that of the dance troupe, that was so effectively executed on the practice field, or on the rehearsal stage, may not be accurately reproduced under game, or performance, conditions.

With this in mind, it would seem fruitful to examine other kinds of activities or states of consciousness that might alter duration estimation as measured in the present research. For example, while many studies have examined the effects of variation in task demands, it would seem that manipulations of emotional experience, in terms of both quality and quantity, might also affect temporal experience. In addition, future research might use the reproduction method to examine the effects of comparable and contrasting tasks within the context of a retrospective paradigm. This would provide for a test of the other half of Zakay's (1989) resource allocation model, which proposes LTM processes at work during retrospective duration estimation.

\section{REFERENCES}

BLOCK, R. A. (1978). Remembered duration: Effects of event and sequence complexity. Memory \& Cognition, 6, 320-326.

BLock, R. A. (1989). Experiencing and remembering time: Affordances, context, and cognition. In I. Levin \& D. Zakay (Eds.), Time and human cognition: A life-span perspective (pp. 333-363). New York: Elsevier.

BLock, R. A. (1990). Models of psychological time. In R. A. Block (Ed.), Cognitive models of psychological time (pp. 1-35). Hillsdale, NJ: Erlbaum.

Brown, S. W. (1985). Time perception and attention: The effects of prospective versus retrospective paradigms and task demands on perceived duration. Perception \& Psychophysics, 38, 115-124.

BuRnside, W. (1971). Judgment of short time intervals while performing mathematical tasks. Perception \& Psychophysics, 9, 404406.

DYER, F. N. (1973). The Stroop phenomenon and its use in the study of perceptual, cognitive, and response processes. Memory \& Cognition, 1, 106-120.

GibBons, J. D. (1976). Nonparametric methods for quantitative analysis. Chicago: Holt, Rinehart \& Winston.

Hicks, R. E., Miller, G. W., Gaes, G., \& Bierman, K. (1977). Concurrent processing demands and the experience of time in passing. American Journal of Psychology, 90, 413-436.

Hicks, R. E., Miller, G. W., \& Kinsbourne, M. (1976). Prospective and retrospective judgments of time as a function of amount of information processed. American Journal of Psychology, 89, 719-730. 
Jensen, A. R., \& Rohwer, W. D., JR. (1966). The Stroop color-word test: A review. Acta Psychologica, 25, 36-93.

Miller, G. W., Hicks, R. E., \& Willette, M. (1978). Effects of concurrent verbal rehearsal and temporal set upon judgments of temporal duration. Acta Psychologica, 42, 173-179.

ORNSTEIN, R. E. (1969). On the experience of time. Harmondsworth, U.K.: Penguin.

POYNTER, W. D. (1983). Duration judgment and the segmentation of experience. Memory \& Cognition, 11, 77-82.

POYNTER, W. D. (1989). Judging the duration of time intervals: A process of remembering segments of experience. In I. Levin \& D. Zakay (Eds.), Time and human cognition: A life-span perspective (pp. 305-331). New York: Elsevier.

Predebon, J. (1988). Remembered duration and perceptual reversals. Acta Psychologica, 69, 157-163.

Sмiтн, N. C. (1969). The effect on time estimation of increasing the complexity of a cognitive task. Journal of General Psychology, 81, 231-235.

Thomas, E. A. C., \& Weaver, W. B. (1975). Cognitive processing and time perception. Perception \& Psychophysics, 17, 363-367.

Toglia, M. P., \& BatTig, W. F. (1978). Handbook of semantic word norms. Hillsdale, NJ: Erlbaum.

ZAKAY, D. (1989). Subjective time and attentional allocation: An integrated model of time estimation. In I. Levin \& D. Zakay (Eds.), Time and human cognition: A life-span perspective (pp. 365-397). New York: Elsevier.

ZAKAY, D. (1990). The evasive art of subjective time measurement: Some methodological dilemmas. In R. A. Block (Ed.), Cognitive models of psychological time (pp. 59-84). Hillsdale, NJ: Erlbaum.

ZAKAY, D. (1993). Time estimation methods-do they influence prospective duration estimates? Perception, 22, 91-101.

ZAKAY, D., \& FALLACH, E. (1984). Immediate and remote time estimation: A comparison. Acta Psychologica, 57, 69-81.

ZaKaY, D., Nitzan, D., \& GliCKSOHN, J. (1983). The influence of task difficulty and external tempo on subjective time estimation. Perception \& Psychophysics, 34, 451-456.

\section{NOTES}

1. The anagrams were created by arbitrarily scrambling the letters of a set of four-letter words sampled from Cluster 8 of the Handbook of Semantic Word Norms (Toglia \& Battig, 1978).

2. The authors want to express their appreciation to reviewer Yoshitaka Nakajima and the editor for their suggestion to examine the results in this fashion.

3. For example, on trials in which subjects were faced with the make $X$ s task during the target interval and anagrams during reproduction, this procedure would generate an expected proportional error equal to $\left[\mathrm{EPE}=\left(\mathrm{MRP}_{\mathrm{MX}} / \mathrm{MRP}_{\mathrm{AG}}\right)-1.0=(.886 / .567)-1.0=+.563\right]$. Also, note that on trials in which subjects performed the same tasks during the target interval and reproduction phase, the $\mathrm{EPE}=0$.

(Manuscript received November 12, 1993; revision accepted for publication June 19,1994 .) 